\title{
Nitrogen Self-Doped Porous Carbon for High-Performance
}

\section{Supercapacitors}

Youning Gong, ${ }^{\dagger}, \|$ Delong Li,$\stackrel{\dagger}{*}$ Qiang Fu, ${ }^{\S}$ Yupeng Zhang ${ }^{*}, \dagger$ and Chunxu Pan ${ }^{*}, \uparrow, \S, \|$

${ }^{\dagger}$ School of Physics and Technology, and MOE Key Laboratory of Artificial Microand Nano-structures, Wuhan University, Wuhan 430072, China

\#Institute of Microscale Optoelectronics, College of Chemistry and Environmental Engineering, Shenzhen University, Shenzhen 518060, China

$\S_{\text {Center for Electron Microscopy, Wuhan University, Wuhan 430072, China }}$

$\|_{\text {Shenzhen Research Institute, Wuhan University, Shenzhen 518057, China }}$

Corresponding Author

*E-mail: ypzhang@szu.edu.cn (Y.Z.).

*E-mail: cxpan@whu.edu.cn (C.P.). 


\section{Electrochemical measurements}

The electrochemical properties of a single electrode were measured in a three-electrode cell system, wherein platinum as the counter electrode and $\mathrm{Hg} / \mathrm{HgO}$ as the reference electrode. The electrolyte was aqueous $\mathrm{KOH}$ solution $(6 \mathrm{M})$. The cyclic voltammetry (CV) and galvanostatic charge/discharge (GCD) experiments were carried out on an electrochemical working station (CHI 760E, Shanghai Chenhua, China). Electrochemical impedance spectroscopy (EIS) was recorded in a frequency range from $0.01 \mathrm{~Hz}$ to $100 \mathrm{~K} \mathrm{~Hz}$ by applying an $\mathrm{AC}$ voltage with the amplitude of 5 $\mathrm{mV}$. The specific electrode capacitance $\left(C_{3 \mathrm{E}}, \mathrm{F} \mathrm{g}^{-1}\right)$ was calculated from GCD profiles by the equation $C_{3 \mathrm{E}}=I_{3 \mathrm{E}} \Delta t_{3 \mathrm{E}} / m_{3 \mathrm{E}} \Delta V_{3 \mathrm{E}}$, in which $I_{3 \mathrm{E}}$ is the constant discharge current, $m_{3 \mathrm{E}}$ is the mass of active material, $\Delta t_{3 \mathrm{E}}$ is the discharging time corresponding to the potential change of $\Delta V_{3 \mathrm{E}}$.

For the CS-HPGC symmetric supercapacitors, CV, GCD and cycling experiments were carried out on the above electrochemical working station and a LAND CT2000 battery testing system. The voltage windows of supercapacitors in $\mathrm{KOH}$ and EMIM TFSI were $0-1.0 \mathrm{~V}$ and $0-3.0 \mathrm{~V}$, respectively. The specific capacitance of a symmetric supercapacitor $\left(C_{\text {cell }}, \mathrm{F} \mathrm{g}^{-1}\right)$ was calculated according to the equation $C_{\text {cell }}=I_{2 \mathrm{E}} \Delta t_{2 \mathrm{E}} / m_{2 \mathrm{E}} \Delta V_{2 \mathrm{E}}$, where $I_{2 \mathrm{E}}$ is the constant discharge current, $m_{2 \mathrm{E}}$ is the total active materials mass on both electrodes, $\Delta t_{2 \mathrm{E}}$ is the discharging time corresponding to the voltage change of $\Delta V_{2 \mathrm{E}}$. The energy density $\left(E, \mathrm{Wh} \mathrm{kg}^{-1}\right)$ and power density $(P, \mathrm{~W}$ $\left.\mathrm{kg}^{-1}\right)$ were calculated by the following equations: $E=C_{\text {cell }} \Delta V_{2 \mathrm{E}}^{2} /(2 \times 3.6)$ and $P=3600 E / \Delta t_{2 \mathrm{E}}$. $^{1,2}$ 


\section{Formation mechanism of the CS-HPGC:}

For a typical synthesis, $\mathrm{K}_{2} \mathrm{FeO}_{4}$ was utilized to provide the activating agent (K) and catalyst (Fe species) to fulfil the synchronous carbonization and graphitization of CS aerogels, which is illustrated in equation (1). The following reactions were proceeded with $\mathrm{KOH}$ activation and Fe catalysis, which do not necessarily take place in a sequential order. ${ }^{3-5}$

$$
4 \mathrm{~K}_{2} \mathrm{FeO}_{4}+10 \mathrm{H}_{2} \mathrm{O} \longrightarrow 8 \mathrm{KOH}+4 \mathrm{Fe}(\mathrm{OH})_{3}+3 \mathrm{O}_{2}
$$

In the $\mathrm{KOH}$ activation, the reactions began with solid-solid reactions and then solid-liquid reactions including the reduction of $\mathrm{K}$ compounds to metallic $\mathrm{K}$, the oxidation of $\mathrm{C}$ to $\mathrm{CO} / \mathrm{CO}_{2}$ and carbonate, and other reactions among various active intermediates. ${ }^{6-10}$ Firstly, $\mathrm{KOH}$ started to dehydrate into $\mathrm{K}_{2} \mathrm{O}$ at $400{ }^{\circ} \mathrm{C}$ (equation (2)) and $\mathrm{C}$ was etched by the produced $\mathrm{H}_{2} \mathrm{O}$ (equation (3)). ${ }^{6} \mathrm{~K}_{2} \mathrm{CO}_{3}$ was also formed at this stage (equation (4)-(5)). Then at $600{ }^{\circ} \mathrm{C}, \mathrm{KOH}$ was completely consumed. ${ }^{7}$

$$
\begin{gathered}
2 \mathrm{KOH} \longrightarrow \mathrm{K}_{2} \mathrm{O}+\mathrm{H}_{2} \mathrm{O} \\
\mathrm{C}+\mathrm{H}_{2} \mathrm{O} \longrightarrow \mathrm{CO}_{+} \mathrm{H}_{2} \\
\mathrm{CO}+\mathrm{H}_{2} \mathrm{O} \longrightarrow \mathrm{CO}_{2}+\mathrm{H}_{2} \\
\mathrm{~K}_{2} \mathrm{O}+\mathrm{CO}_{2} \longrightarrow \mathrm{K}_{2} \mathrm{CO}_{3}
\end{gathered}
$$

When above $700{ }^{\circ} \mathrm{C}, \mathrm{K}_{2} \mathrm{CO}_{3}$ started to decompose into $\mathrm{K}_{2} \mathrm{O}$ and $\mathrm{CO}_{2}$, and was completely consumed at $800{ }^{\circ} \mathrm{C}$ (eqn (6)). ${ }^{8}$ Additionally, the produced $\mathrm{CO}_{2}$ and $\mathrm{K}$ compounds could further react with carbon over $700{ }^{\circ} \mathrm{C}$ (eqn (7)-(9))..$^{9,10}$ The carbon lattices were finally expanded irreversibly and formed a high microporosity during the physical and chemical activations.

$$
\begin{gathered}
\mathrm{K}_{2} \mathrm{CO}_{3} \longrightarrow \mathrm{K}_{2} \mathrm{O}+\mathrm{CO}_{2} \\
\mathrm{CO}_{2}+\mathrm{C} \longrightarrow 2 \mathrm{CO} \\
\mathrm{K}_{2} \mathrm{CO}_{3}+2 \mathrm{C} \longrightarrow 2 \mathrm{~K}+3 \mathrm{CO} \\
\mathrm{K}_{2} \mathrm{O}+\mathrm{C} \longrightarrow 2 \mathrm{~K}+\mathrm{CO}
\end{gathered}
$$


For the graphitization, amorphous $\mathrm{Fe}$ compounds $\left(\mathrm{Fe}(\mathrm{OH})_{3}, \mathrm{FeO}(\mathrm{OH})\right)$ were first transformed to $\mathrm{Fe}_{2} \mathrm{O}_{3}$ (equation (10)), and then were reduced to $\mathrm{Fe}_{3} \mathrm{O}_{4}$ (equation (11)). ${ }^{5}$ The $\mathrm{Fe}_{3} \mathrm{O}_{4}$ was further reduced to metallic $\mathrm{Fe}$ (equation (12)), which finally act as the catalyst which converted the amorphous carbon into graphitic carbon. ${ }^{11,12}$

$$
\begin{gathered}
\mathrm{Fe}(\mathrm{OH})_{3} \longrightarrow \mathrm{FeO}(\mathrm{OH}) \longrightarrow \mathrm{Fe}_{2} \mathrm{O}_{3} \\
3 \mathrm{Fe}_{2} \mathrm{O}_{3}+\left(\mathrm{H}_{2}, \mathrm{C}, \mathrm{CO}\right) \longrightarrow 2 \mathrm{Fe}_{3} \mathrm{O}_{4}+\left(\mathrm{H}_{2} \mathrm{O}, \mathrm{CO}, \mathrm{CO}_{2}\right) \\
\mathrm{Fe}_{3} \mathrm{O}_{4}+4\left(\mathrm{H}_{2}, \mathrm{C}, \mathrm{CO}\right) \longrightarrow 3 \mathrm{Fe}+4\left(\mathrm{H}_{2} \mathrm{O}, \mathrm{CO}, \mathrm{CO}_{2}\right)
\end{gathered}
$$



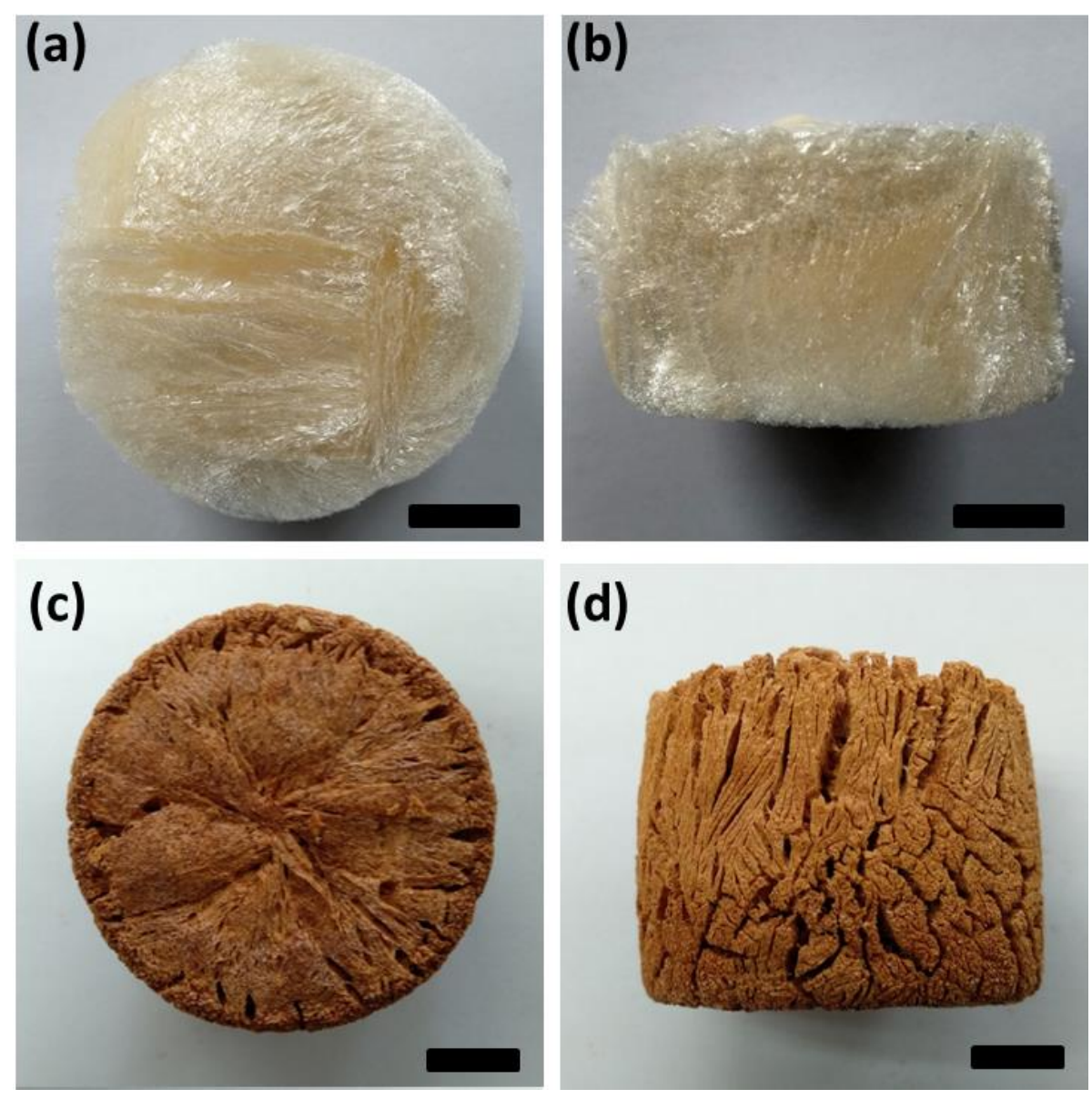

Figure S1. Photographs of the chitosan-derived aerogels: $(a, b)$ CS-PC, (c, d) CS-HPGC. Scale bars: $1 \mathrm{~cm}$. 

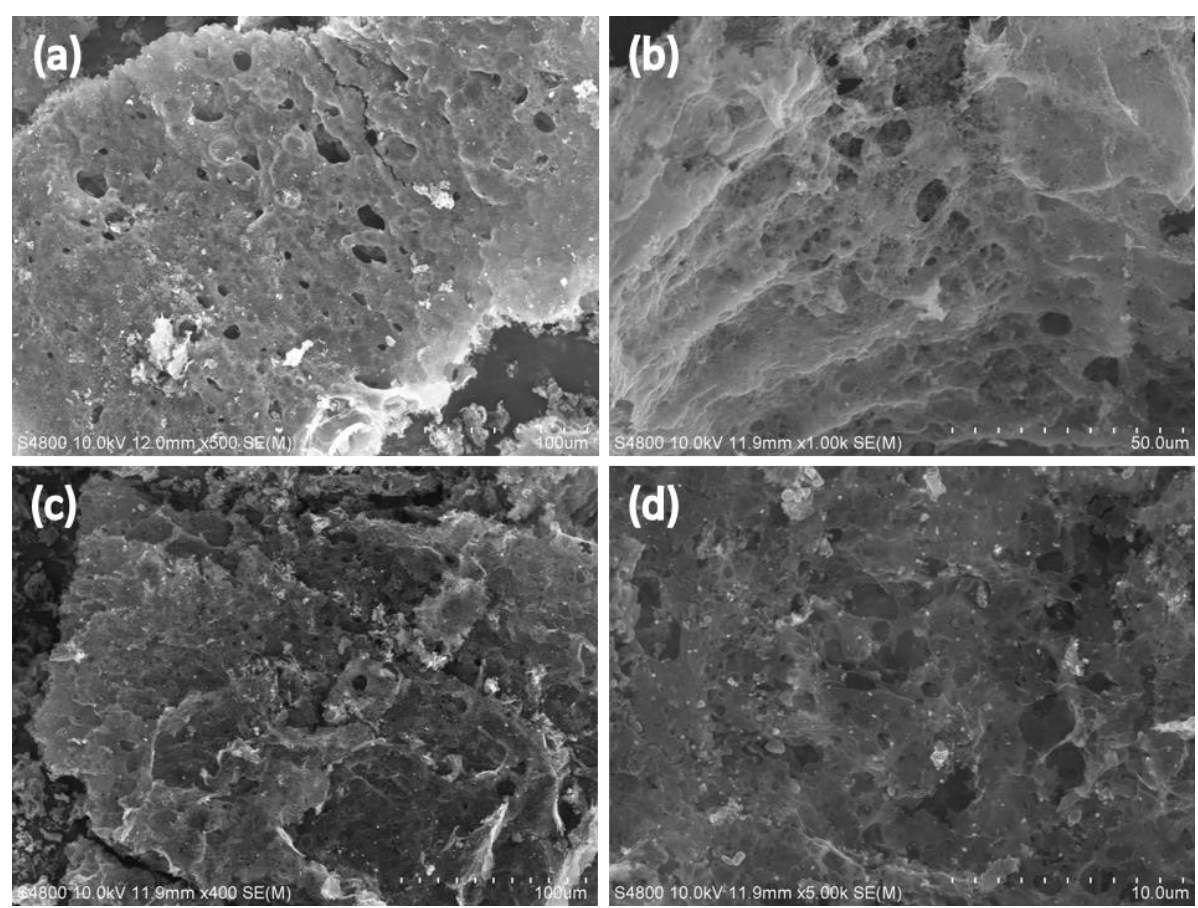

Figure S2. SEM morphologies: (a, b) CS-HPGC-1 sample, and (c, d) CS-HPGC-3 sample.
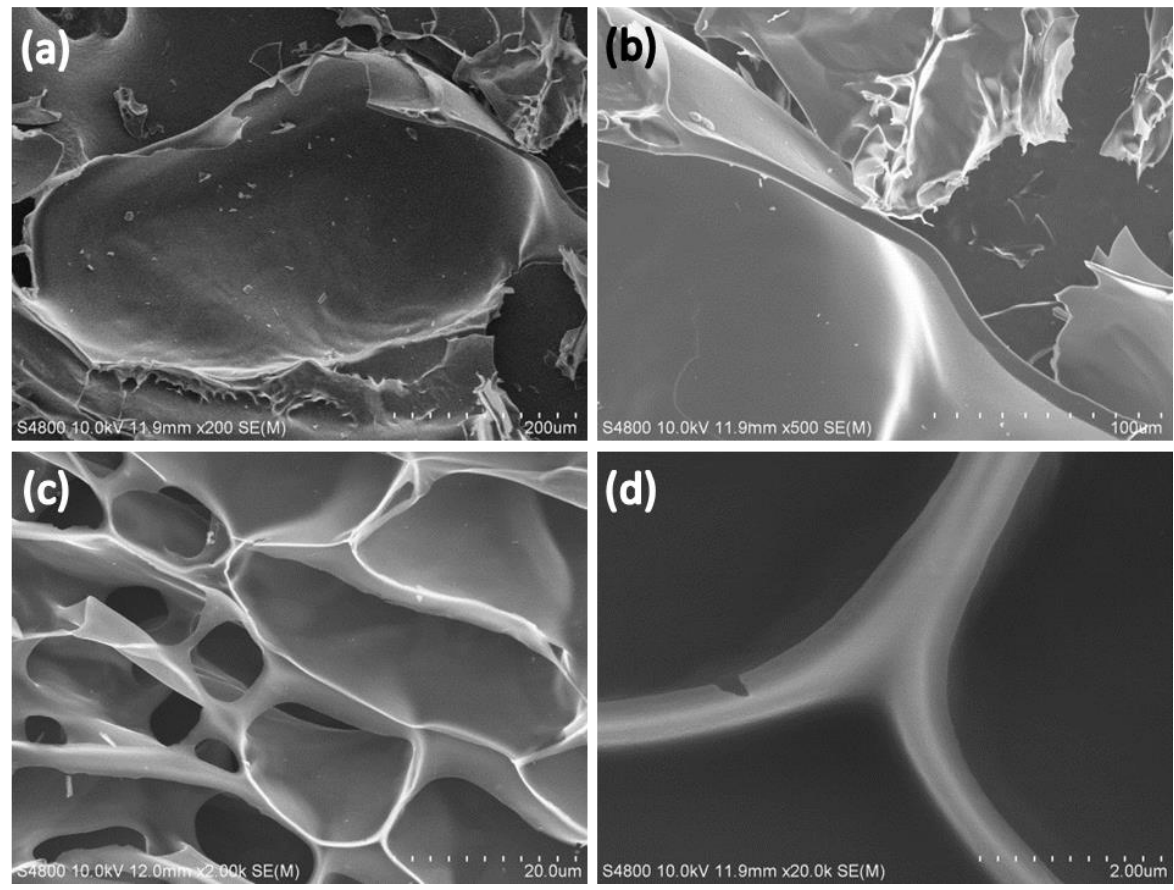

Figure S3. SEM morphologies of the CS-PC sample: (a, c) low magnification, (b, d) high magnification. 
(a)

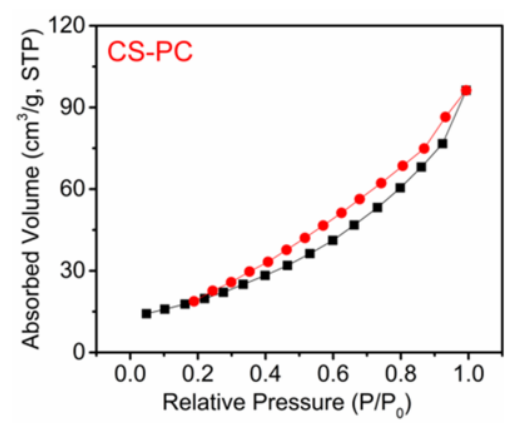

(c)

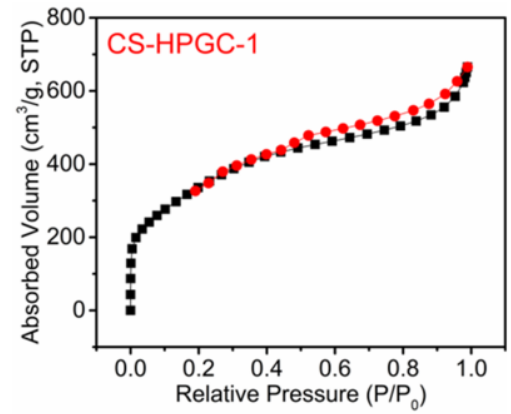

(e)

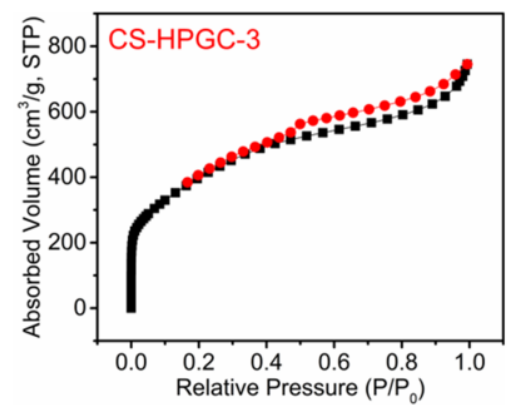

(b)

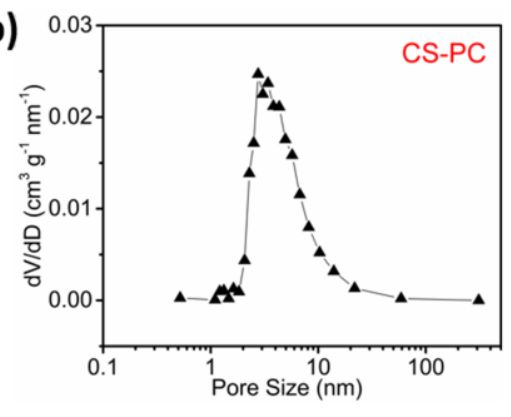

(d)

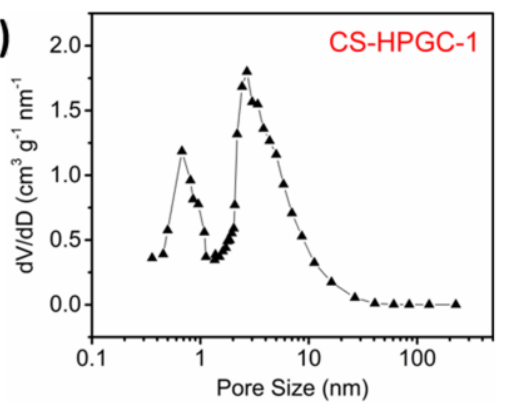

(f)

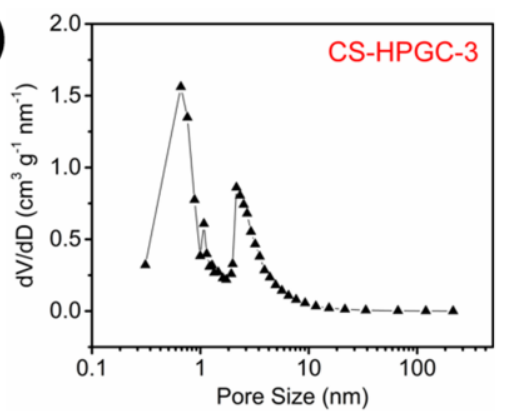

Figure S4. $\mathrm{N}_{2}$ adsorption-desorption isotherms and pore size distributions of CS-derived carbon samples. 

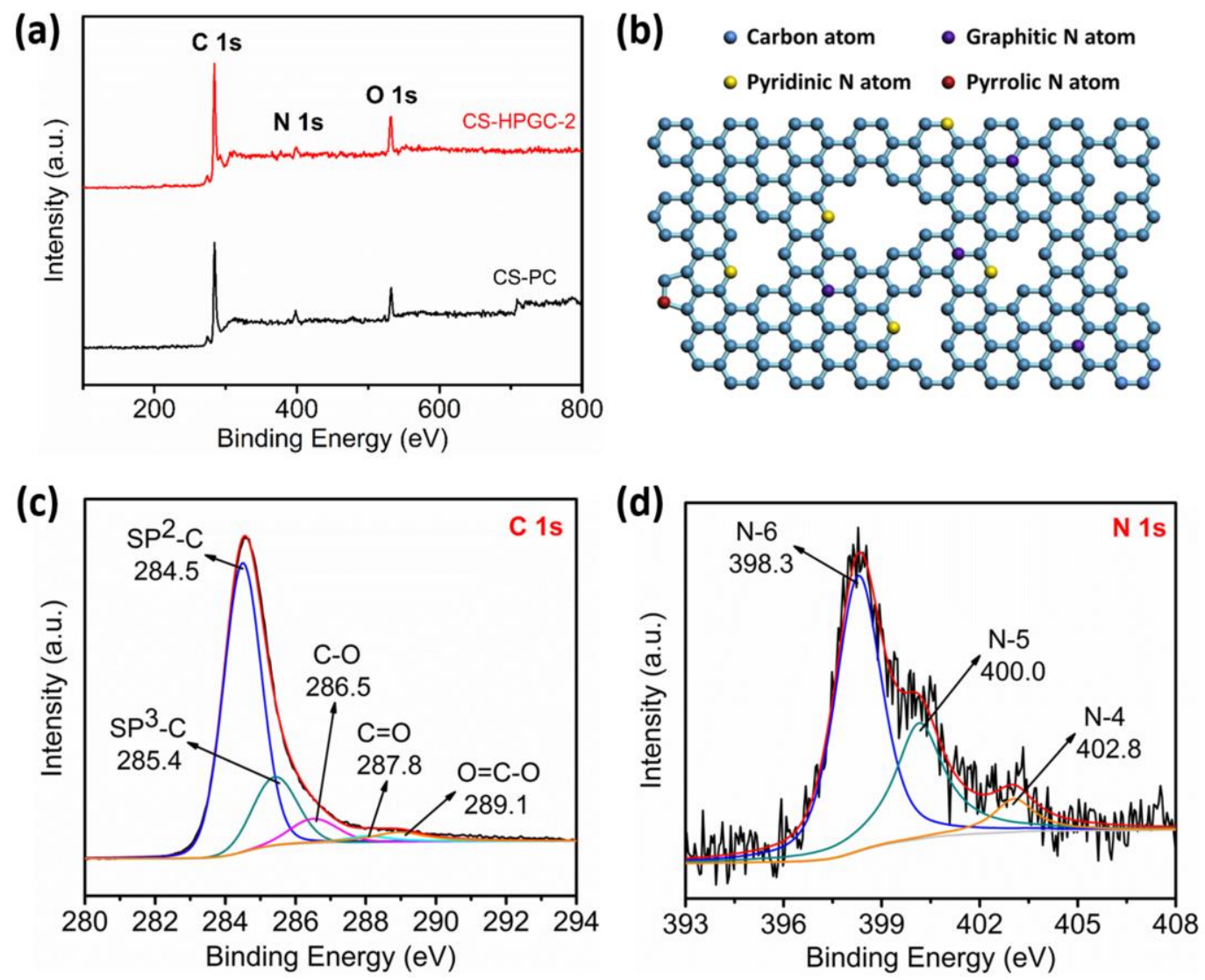

Figure S5. (a) XPS survey spectra of the CS-derived carbon samples; (b) bonding configurations of nitrogen atoms in CS-derived carbon; (c) C 1s and (d) N 1s high-resolution XPS spectra of the CS-PC sample. 
(a)

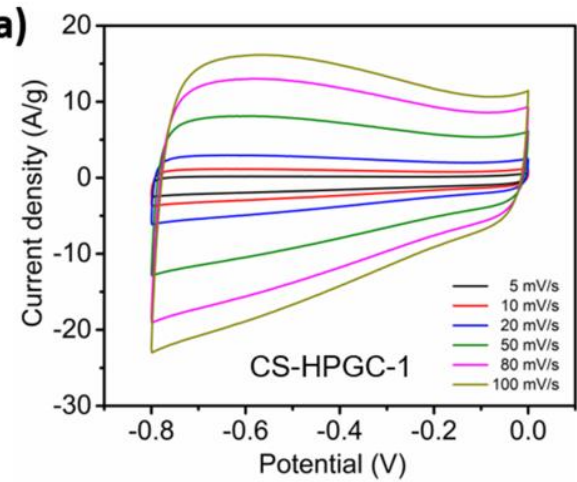

(c)

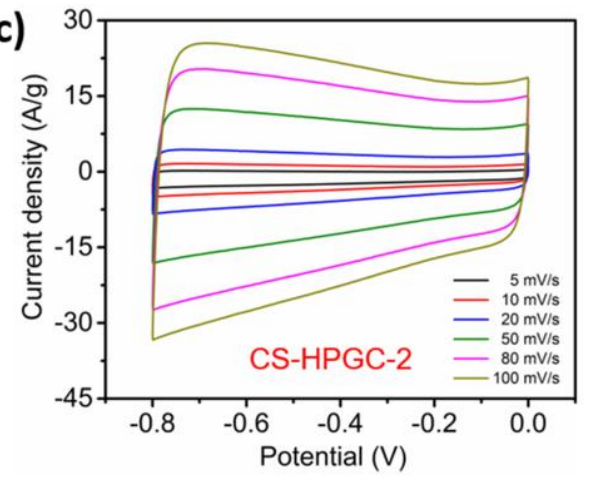

(e)

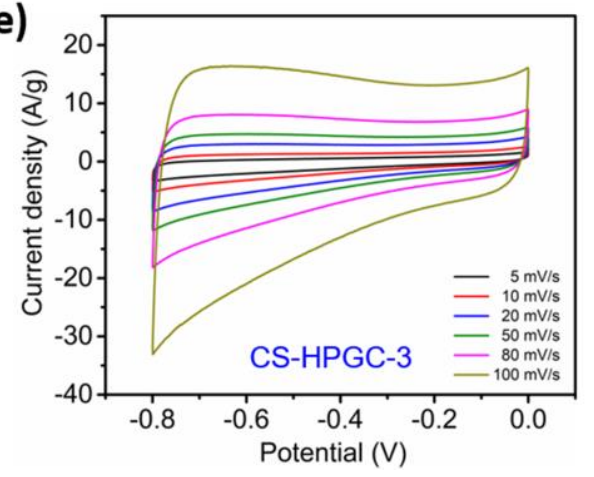

(b)

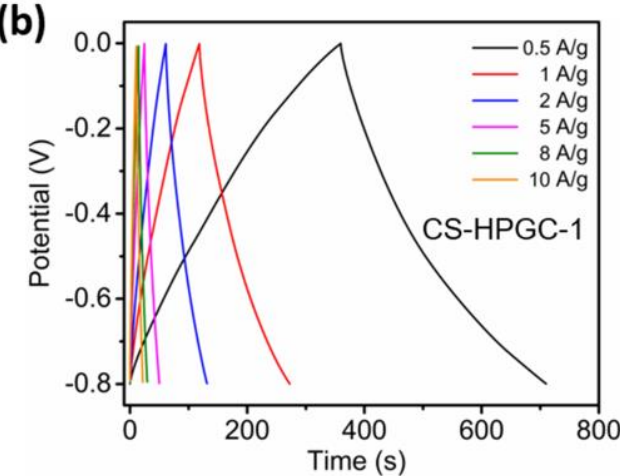

(d)
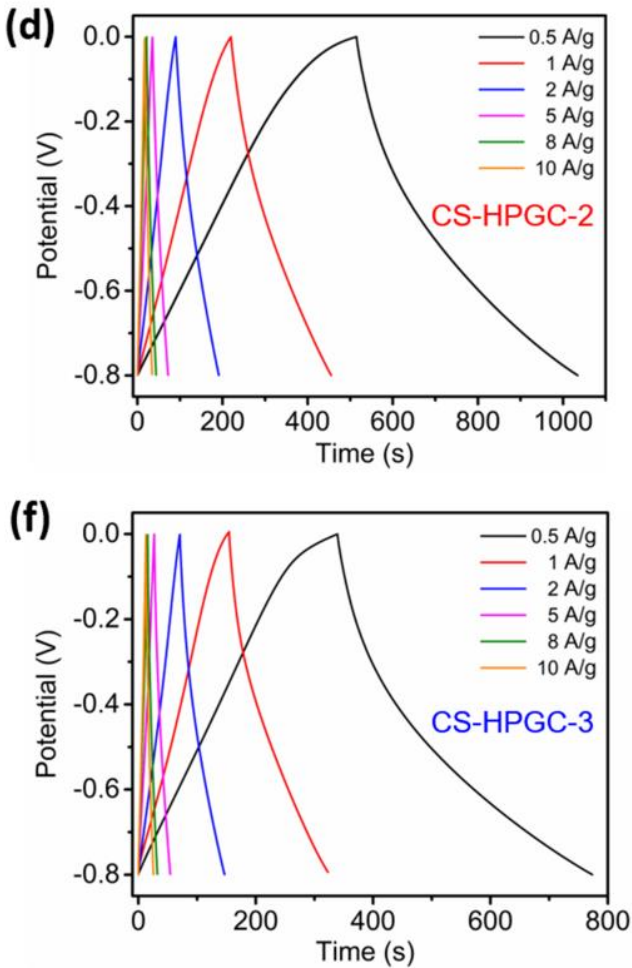

Figure S6. CV and GCD profiles of the CS-HPGC electrodes: (a, b) CS-HPGC-1; (c, d) CS-HPGC-2; (e, f) CS-HPGC-3. 
Table S1 Chemical compositions of CS-HPGC-2 sample in different etching depths

\begin{tabular}{cccc}
\hline \multirow{2}{*}{$\begin{array}{c}\text { Depth } \\
(\mathbf{n m})\end{array}$} & \multicolumn{3}{c}{ Elemental content (at\%) } \\
\cline { 2 - 4 } & $\mathbf{C}$ & $\mathbf{O}$ & $\mathbf{N}$ \\
\hline 0 & 83.3 & 10.8 & 5.9 \\
10 & 84.0 & 10.4 & 5.6 \\
20 & 83.2 & 10.8 & 6.0 \\
30 & 82.4 & 11.9 & 5.7 \\
Average & 83.2 & 11.0 & 5.8 \\
\hline
\end{tabular}


Table S2 Comparison of various biomass-derived carbon materials by different activation methods for supercapacitor application

\begin{tabular}{|c|c|c|c|c|c|c|}
\hline Materials & $\begin{array}{l}\text { Activation } \\
\text { method }\end{array}$ & $\begin{array}{c}\text { Specific } \\
\text { surface area } \\
\left(\mathbf{m}^{2} \mathbf{g}^{-1}\right)\end{array}$ & $\begin{array}{c}\text { Pore } \\
\text { volume } \\
\left(\mathrm{cm}^{3} \mathrm{~g}^{-1}\right)\end{array}$ & Electrolyte & $\begin{array}{c}\text { Specific } \\
\text { capacitance } \\
\left(\mathbf{F ~ g ~}^{-1}\right)\end{array}$ & Ref. \\
\hline $\begin{array}{c}\text { Coffee } \\
\text { Endocarp }\end{array}$ & $\begin{array}{c}\mathrm{CO}_{2} \\
\text { activation }\end{array}$ & 1050 & 0.5 & $1 \mathrm{M} \mathrm{H}_{2} \mathrm{SO}_{4}$ & $\begin{array}{c}176 \\
(10 \mathrm{~mA})\end{array}$ & 13 \\
\hline $\begin{array}{l}\text { Rubber } \\
\text { Wood } \\
\text { Sawdust }\end{array}$ & $\begin{array}{c}\mathrm{CO}_{2} \\
\text { activation }\end{array}$ & 913 & 0.61 & $1 \mathrm{M} \mathrm{H}_{2} \mathrm{SO}_{4}$ & $\begin{array}{c}138 \\
\left(10 \mathrm{~mA} \mathrm{~cm}^{-2}\right)\end{array}$ & 14 \\
\hline Oil Palm & $\begin{array}{l}\mathrm{CO}_{2} / \mathrm{KOH} \\
\text { activation }\end{array}$ & 1704 & 0.889 & $\mathrm{H}_{2} \mathrm{SO}_{4}$ & $\begin{array}{c}150 \\
\left(10 \mathrm{~mA} \mathrm{~cm}^{-2}\right)\end{array}$ & 15 \\
\hline $\begin{array}{l}\text { Waste } \\
\text { Paper }\end{array}$ & $\begin{array}{c}\mathrm{KOH} \\
\text { activation }\end{array}$ & 416 & 0.225 & $6 \mathrm{M} \mathrm{KOH}$ & $\begin{array}{c}160 \\
\left(1 \mathrm{~mA} \mathrm{~cm}^{-2}\right)\end{array}$ & 16 \\
\hline $\begin{array}{l}\text { Cow } \\
\text { Dung }\end{array}$ & $\begin{array}{c}\mathrm{KOH} \\
\text { activation }\end{array}$ & 1984 & 0.91 & $\begin{array}{c}1.0 \mathrm{M} \\
\mathrm{Et}_{4} \mathrm{NBF}_{4}\end{array}$ & $\begin{array}{c}124 \\
\left(0.1 \mathrm{~A} \mathrm{~g}^{-1}\right)\end{array}$ & 17 \\
\hline $\begin{array}{l}\text { Willow } \\
\text { Catkins }\end{array}$ & $\begin{array}{c}\mathrm{KOH} \\
\text { activation }\end{array}$ & 1586 & 0.78 & $6 \mathrm{M} \mathrm{KOH}$ & $\begin{array}{c}253 \\
\left(0.1 \mathrm{Ag}^{-1}\right)\end{array}$ & 18 \\
\hline $\begin{array}{c}\text { Sunflower } \\
\text { Seed } \\
\text { Shell }\end{array}$ & $\begin{array}{c}\mathrm{KOH} \\
\text { activation }\end{array}$ & 2585 & 1.41 & $\begin{array}{c}30 \mathrm{wt} \% \\
\mathrm{KOH}\end{array}$ & $\begin{array}{c}311 \\
\left(0.25 \mathrm{~A} \mathrm{~g}^{-1}\right)\end{array}$ & 19 \\
\hline $\begin{array}{l}\text { Human } \\
\text { Hair }\end{array}$ & $\begin{array}{c}\mathrm{KOH} \\
\text { activation }\end{array}$ & 1306 & 0.9 & $6 \mathrm{M} \mathrm{KOH}$ & $\begin{array}{c}340 \\
\left(1.0 \mathrm{Ag}^{-1}\right)\end{array}$ & 20 \\
\hline $\begin{array}{l}\text { Fallen } \\
\text { Leave }\end{array}$ & $\begin{array}{c}\mathrm{KOH} / \mathrm{K}_{2} \mathrm{CO}_{3} \\
\text { activation }\end{array}$ & 2869 & 1.598 & $6 \mathrm{M} \mathrm{KOH}$ & $\begin{array}{c}242 \\
\left(0.3 \mathrm{Ag}^{-1}\right)\end{array}$ & 21 \\
\hline
\end{tabular}




\begin{tabular}{|c|c|c|c|c|c|c|}
\hline $\begin{array}{l}\text { Hemi- } \\
\text { cellulose }\end{array}$ & $\begin{array}{l}\text { Hydrothermal } \\
\text { carbonization } \\
\text { / KOH } \\
\text { activation }\end{array}$ & 2200 & 1.0 & $\begin{array}{c}0.5 \mathrm{M} \\
\mathrm{H}_{2} \mathrm{SO}_{4}\end{array}$ & $\begin{array}{c}300 \\
\left(0.25 \mathrm{Ag}^{-1}\right)\end{array}$ & 22 \\
\hline $\begin{array}{c}\text { Bamboo } \\
\text { waste }\end{array}$ & $\begin{array}{l}\text { Hydrothermal } \\
\text { carbonization } \\
\text { / } \mathrm{KOH} \\
\text { activation }\end{array}$ & 1472 & - & $6 \mathrm{M} \mathrm{KOH}$ & $\begin{array}{c}301 \\
\left(0.1 \mathrm{Ag}^{-1}\right)\end{array}$ & 23 \\
\hline Chitosan & $\begin{array}{c}\mathrm{K}_{2} \mathrm{FeO}_{4} \\
\text { activation }\end{array}$ & 1770 & 1.15 & $6 \mathrm{M} \mathrm{KOH}$ & $\begin{array}{c}332.3\left(0.5 \mathrm{~A} \mathrm{~g}^{-1}\right) \\
294.1\left(1.0 \mathrm{~A} \mathrm{~g}^{-1}\right) \\
217.5\left(10 \mathrm{~A} \mathrm{~g}^{-1}\right)\end{array}$ & $\begin{array}{l}\text { This } \\
\text { work }\end{array}$ \\
\hline
\end{tabular}

\section{References}

[1] Qu, D.; Shi, H. Studies of Activated Carbons Used in Double-Layer Capacitors. J. Power Sources 1998, 74, 99-107.

[2] Wang, Y.; Song, Y.; Xia, Y. Electrochemical Capacitors: Mechanism, Materials, Systems, Characterization and Applications. Chem. Soc. Rev. 2016, 45, 5925-5950.

[3] Wang, J.; Kaskel, S. KOH Activation of Carbon-Based Materials for Energy Storage. J. Mater. Chem. 2012, 22, 23710-23725.

[4] Shen, Y. Carbothermal Synthesis of Metal-functionalized Nanostructures for Energy and Environmental Applications. J. Mater. Chem. A 2015, 3, 13114-13188.

[5] Liu, W. J.; Tian, K.; He, Y. R.; Jiang, H.; Yu, H. Q. High-Yield Harvest of Nanofibers/Mesoporous Carbon Composite by Pyrolysis of Waste Biomass and Its Application for High Durability Electrochemical Energy Storage. Environ. Sci. Technol. 2014, 48, 13951-13959.

[6] Otaw, T.; Tanibata R.; Itoh M. Production and Adsorption Characteristics of MAXSORB: High-Surface-Area Active Carbon. Gas Separation \& Purification 1993, 7, 241-245.

[7] Díaz-Terán, J.; Nevskaia, D. M.; Fierro, J. L. G.; López-Peinado, A. J.; Jerez, A. Study of Chemical Activation Process of a Lignocellulosic Material with KOH by XPS and XRD. Micropor. Mesopor. Mat. 2003, 60, 173-181.

[8] Illán-Gómez, M. J.; García-García, A.; Salinas-Martínez de Lecea, C.; Linares-Solano, A. Activated Carbons from Spanish Coals. 2. Chemical Activation. Energ. Fuel. 1996, 10, 1108-1114.

[9] McKee, D. W. Gasification of Graphite in Carbon Dioxide and Water Vapor-The Catalytic Effects of Alkali Metal Salts. Carbon 1982, 20, 59-66.

[10]Teng, H.; Hsu, L. -Y. High-Porosity Carbons Prepared from Bituminous Coal with Potassium Hydroxide Activation. Ind. Eng. Chem. Res. 1999, 38, 2947-2953. 
[11]Maldonado-Hódar, F. J.; Moreno-Castilla, C.; Rivera-Utrilla, J.; Hanzawa, Y.; Yamada, Y. Catalytic Graphitization of Carbon Aerogels by Transition Metals Langmuir 2000, 16, 4367-4373.

[12] Sevilla, M.; Fuertes, A. B. Catalytic Graphitization of Templated Mesoporous Carbons. Carbon 2006, 44, 468-474.

[13]Nabais, J. M. V.; Teixeira. J. G.; Almeida, I. Development of Easy Made Low Cost Bindless Monolithic Electrodes from Biomass with Controlled Properties to be Used as Electrochemical Capacitors. Bioresource Technol. 2011, 102, 2781-2787.

[14]Taer, E.; Deraman, M.; Talib, I. A.; Awitdrus, A.; Hashmi, S. A.; Umar, A. A. Preparation of a Highly Porous Binderless Activated Carbon Monolith from Rubber Wood Sawdust by a Multi-Step Activation Process for Application in Supercapacitors. Int. J. Electrochem. Sci. 2011, 6, 3301-3315.

[15]Farma, R.; Deraman, M.; Awitdrus, A.; Talib, I. A.; Taer, E.; Basri, N. H.; Manjunatha, J. G.; Ishak, M. M.; Dollah, B. N. M.; Hashmi, S. A. Preparation of Highly Porous Binderless Activated Carbon Electrodes from Fibres of Oil Palm Empty Fruit Bunches for Application in Supercapacitors. Bioresource Technol. 2013, 132, 254-261.

[16]Kalpana, D.; Cho, S. H.; Lee, S. B.; Lee, Y. S.; Misra, R.; Renganathan, N.G. Recycled Waste Paper-A New Source of Raw Material for Electric Double-Layer Capacitors. J. Power Sources 2009, 190, 597-591.

[17]Bhattacharjya, D.; Yu, J. S. Activated Carbon Made from Cow Dung as Electrode Material for Electrochemical Double Layer Capacitor. J. Power Sources 2014, 262, 224-231.

[18]Wang, K.; Zhao, N.; Lei, S.; Yan, R.; Tian, X.; Wang, J.; Song, Y.; Xu, D.; Guo, Q.; Liu, L. Promising Biomass-Based Activated Carbons Derived from Willow Catkins for High Performance Supercapacitors. Electrochim. Acta 2015, 166, $1-11$.

[19]Li, X.; Xing, W.; Zhuo, S.; Zhou, J.; Li, F.; Qiao, S. Z.; Lu, G. Q. Preparation of Capacitor's Electrode from Sunflower Seed Shell. Bioresource Technol. 2011, 102, 1118-1123.

[20]Qian, W.; Sun, F.; Xu, Y.; Qiu, L.; Liu, C.; Wang, S.; Yan, F. Human Hair-Derived Carbon Flakes for Electrochemical Supercapacitors. Energy Environ. Sci. 2014, 7, 379-386.

[21]Li, Y. T.; Pi, Y. T.; Lu, L. M.; Xu, S. H.; Ren, T. Z. Hierarchical Porous Active Carbon from Fallen Leaves by Synergy of $\mathrm{K}_{2} \mathrm{CO}_{3}$ and Their Supercapacitor Performance. J. Power Sources 2015, 299, 519-528.

[22]Falco, C.; Sieben, J. M.; Brun, N.; Sevilla, M.; Mauelen, T. V. D.; Morallón, E.; Amorós, D. C.; Titirici, M. M. Hydrothermal Carbons from Hemicellulose-Derived Aqueous Hydrolysis Products as Electrode Materials for Supercapacitors. ChemSusChem 2013, 6, 374-382. 
[23]Tian, W.; Gao, Q.; Tan, Y.; Yang, K.; Zhu, L.; Yang, C.; Zhang, H. Bio-Inspired Beehive-Like Hierarchical Nanoporous Carbon Derived from Bamboo-Based Industrial Byproduct as a High Performance Supercapacitor Electrode Material. J. Mater. Chem. A 2015, 3, 5656-5664. 